\title{
The role of G-CSF in recurrent implantation failure: A randomized double blind placebo control trial
}

\author{
Fatemeh Davari-tanha' M.D., Ensieh Shahrokh Tehraninejad ${ }^{2}$ M.D., Mohadese Ghazi ${ }^{1}$ M.D., Zahra \\ Shahraki ${ }^{1}$ M.D.
}

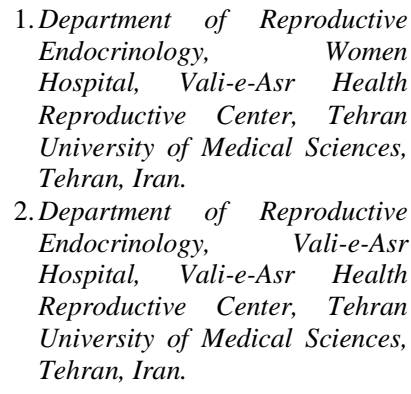

2.Department of Reproductive Endocrinology, Vali-e-Asr Hospital, Vali-e-Asr Health Reproductive Center, Tehran University of Medical Sciences, Tehran, Iran

Corresponding Author:

Fatemeh Davari Tanha, Department of reproductive Endocrinology, Women Hospital, North Ostad Nejatollahi, Pole Karimkhan Zand, Tehran, Iran.

Tel: (98) 2188800115

Email: fatedavtanha@gmail.com

Received: 11 January 2016 Revised: 11 June 2016 Accepted: 28 September 2016

\begin{abstract}
Background: Recurrent implantation failure (RIF) is the absence of implantation after three consecutive In Vitro Fertilization (IVF) cycles with transferring at least four good quality embryos in a minimum of three fresh or frozen cycles in a woman under 40 years. The definition and management of RIF is under constant scrutiny.

Objective: To investigate the effects of Granulocyte colony stimulating factor (GCSF) on RIF, pregnancy rate, abortion rate and implantation rates.

Materials and Methods: A double blind placebo controlled randomized trial was conducted at two tertiary university based hospitals. One hundred patients with the history of RIF from December 2011 until January 2014 were recruited in the study. G-CSF $300 \mu \mathrm{g} / 1 \mathrm{ml}$ was administered at the day of oocyte puncture or day of progesterone administration of FET cycle. Forty patients were recruited at G-CSF group, 40 in saline and 20 in placebo group.

Results: The mean age for whole study group was 35.3 \pm 4.2 yrs (G-CSF 35.5 \pm 4.32 , saline $35.3 \pm 3.98$, placebo $35.4 \pm 4.01$, respectively). Seventeen patients had a positive pregnancy test after embryo transfer [10 (25\%) in G-CSF; $5(12.5 \%)$ in saline; and 2 $(10 \%)$ in placebo group]. The mean of abortion rates was $17.6 \%$ (3), two of them in G-CSF, one in saline group. The implantation rate was $12.3 \%$ in G-CSF, $6.1 \%$ in saline and $4.7 \%$ in placebo group.

Conclusion: G-CSF may increase chemical pregnancy and implantation rate in patients with recurrent implantation failure but clinical pregnancy rate and abortion rate was unaffected.
\end{abstract}

Key words: Implantation, Failure, G-CSF, Intrauterine, Pregnancy rate.

Registration ID in IRCT: RCT201108212576N5

\section{Introduction}

$\mathrm{R}$ ecurrent implantation failure (RIF) is defined as failure to achieve pregnancy after in vitro fertilization (IVF) or intracytoplasmic sperm injection, after transferring at least four good quality embryos in at least three fresh or frozen cycles in women younger than forty years old (1). Repetitive implantation failure is an iatrogenic event that is the consequence of embryo or uterine factors $(2,3)$. RIF is different from IVF failure. IVF failure is failure to achieve pregnancy due to poor responder to ovarian induction, absence of good quality embryo, advanced maternal age and finally uterine factors $(4,5)$.

Nowadays RIF is defined basically as implantation failure due to uterine factors. Actually RIF is a subgroup of IVF failure patients who have good quality embryo and age $<40$ but have failure to achieve pregnancy (5). Mullerian anomalies like septate uterus can change endometrial receptivity not only due to disturbance of uterine cavity but also to the inadequate blood supply to the septum (6). Abortion rate after IVF in septate uterus was reported $80 \%$ but after hysteroscopic resection of septum decreased to $30 \%$. Submucosal myoma increases uterine contractility and changes cytokine profile; also it leads to abnormal vascularization and chronic endometiritis. These women have decreased implantation rate. Resection of submucosal myoma hysteroscopically results to double clinical pregnancy rates (7). Also resection of endometrial polyps resulted to more clinical pregnancy rate in Intra Uterine insemination (IUI) cycles (8).

Besides the submucosal myoma and polyp, intrauterine adhesion either after dilatation \& curettage (D\&C) in gravid uterus for abortion 
or after intrauterine infection in nongravid uterus may interfere with successful implantation (9). On the other hand adenomyosis which affects the junctional zone of uterus is more hazardous for implantation than intramural myoma which is far from implantation site (10). Moreover hydrosalpinx removal results to improve pregnancy outcome after IVF (16\% vs. $28.6 \%$ live birth rate) $(11,12)$.

However the immunological factors are considered critical for embryo implantation, there are much conflicting evidence on the value of immunological treatment in patients with RIF $(13,14)$. G-CSF is a hematopoietic specific cytokine produced by bone marrow cells, stromal cells, fibroblasts and macrophages. G-CSF increases phagocytosis and oxidative process which is necessary for implantation (15). Some of nonhematopoetic cell types, including endothelial, placenta, trophoblastic and granolousa luteina cells contain G-CSF receptor (14). Moreover GCSF appears to affect endometrial expression of genes critical for the implantation process, such as endometrial vascular remodeling, local immune modulation and cellular adhesions mechanisms (15).

G-CSF contributes to successful reproduction by increasing implantation and promoting endometrial thickness. Nevertheless many cases of RIF remain unexplained and several etiological factors including immune dysfunction or alloimmune response are proposed; in animal models, GCSF showed a marked anti abortive activity (16). G-CSF play roles in increasing endometrial thickness and decreasing recurrent abortion $(6,17-19)$.

The aim of the present study was the evaluation of G-CSF effects on patients with RIF regarding to pregnancy, abortion and implantation rates.

\section{Materials and methods}

In a randomized double blind placebo controlled clinical trial 100 patients with recurrent implantation failure were included from two tertiary university based hospitals of Tehran University of Medical Sciences from December 2011 until January 2014 in this study. The study was approved by the ethical committee of Tehran University of Medical Sciences with the project code of 14967. All eligible patients signed informed consent before participation in the study. This study was a three-arm randomized clinical trial. Randomization was performed by a computergenerated randomization block table. Randomization cards were offered to the patients by a nurse who was blinded to the study groups. Patients and clinician were blinded regarding the study groups (Figure 1).

Embryo transfer was done as frozen and fresh cycles. Nine patients underwent FET cycle (four patients in G-CSF; 3 patients in saline and two in placebo group) and 91 patients underwent fresh cycle. Inclusion criteria were all patients with RIF under the age of $40 \mathrm{yr}$ old (mean age $=35.3 \pm 4.2 \mathrm{yr}$ ). RIF was defined as history of three times implantation failure when there was history of transferring at least four good quality embryos without uterine or thrombophilic factors. Women with history of renal disease, sickle cell disease or malignancy or sensitivity of GCSF were excluded from study. Gonal-F/HMG was prescribed at a dosage of 150-225 $U$ per day on the second day of menstruation cycle. The ovarian response was evaluated by transvaginal sonography and the need for additional dose was determined according it.

Recombinant hCG was injected when there were at least 3 follicles above $18 \mathrm{~mm}$, and oocyte retrieval was performed $36 \mathrm{hr}$ after injection by transvaginal ultrasound guidance. The G-CSF used was Nupogen $(300 \mu \mathrm{g} / \mathrm{ml}$, Filgrastim; Amgen). At the time of oocyte retrieval one $\mathrm{ml}$ of G-CSF or normal saline was administered by a Trans cervical Cook catheter for embryo transfer slowly into uterine cavity. For placebo group a catheter pass through the cervix without any injection. In FET cycle intervention was scheduled at the day of starting progesterone. Chemical pregnancy was defined as positive $\beta$-hCG two weeks after embryo transfer. Clinical pregnancy was assessed by visualizing gestational sac at transvaginal sonogram three weeks after embryo transfer.

Implantation rates were defined as number of gestational sac four weeks after embryo transfer based on the number of embryos transferred. The good quality embryo at day three was an 8 cells embryo with $<15 \%$ fragmentation or at day five an expanded blastocysts with at least grade $B$ trophoectoderm and inner cell mass. 


\section{Statistical analysis}

All analysis conducted by Statistical Package for the Social Sciences, version 16.0, SPSS Inc, Chicago, Illinois, USA (SPSS 16). For continuous variables, statistical significance was assessed by the use of the two tailed student's t-test for unpaired data. Fisher exact test and $\chi^{2}$ were used when appropriate for discontinuous variables. $p<0.05$ was considered significant.

\section{Results}

The demographic data and baseline characteristics of patients are shown in table I. No differences were found in three groups of patients for the women's age, number of pervious IVF failures, Body Mass Index (BMI), and the number of good quality embryo transfer. The mean follicle stimulating hormone (FSH) level was 7.36 \pm 3.64 (G-CSF $7.98 \pm 4.21$, saline $7.22 \pm 2.98$, and placebo $7.41 \pm 2.51$ ). The mean of endometrial thickness at the day of hCG trigger was $10.23 \pm 2.51 \mathrm{~mm}$ (G-CSF 9.98 \pm 3.61 , saline $10.35 \pm 1.45$, and placebo 10.01 \pm 2.59 ).
There were no significant differences in three groups. Seventeen patients had a positive $\beta$-hCG titer after embryo transfer (10 in G-CSF group, 5 in saline and 2 in placebo group). Fourteen patients established clinical pregnancy: in G-CSF $25 \%$ (8 of 40 patients); in saline group $12.5 \%$ (5 of 40 patients) and in placebo group $10 \%$ (2 of 20 patients). The difference of chemical pregnancy was significant for G-CSF group comparing to saline and placebo group $(p<0.04)$ but the difference between saline and placebo group was not significant $(p<0.15)$.

There were three spontaneous abortion, two out of ten in G-CSF group and one out of five in saline group $(p=0.06)$. Also 210 embryos were transferred during study. The implantation rate was $12.3 \%$ (10 of 81 ) in GCSF; $6.1 \%$ (5 of 87 ) in saline and $4.7 \%$ (2 of 42) in placebo group. The implantation rate was statistically different for G-CSF regarding saline and placebo group $(p=0.04)$. The quality of embryo in three groups was not statistically different but there were more grade A embryos in placebo group.

Table I. Demographic characteristics of participants in three groups (G-CSF, saline, placebo) of RIF patients

\begin{tabular}{|c|c|c|c|c|}
\hline Variable & G-CSF & Saline & Placebo & p-value \\
\hline Age (y) & $35.5 \pm 4.32$ & $35.3 \pm 3.98$ & $35.4 \pm 4.01$ & 0.33 \\
\hline BMI (kg/m2) & $25.2 \pm 1.8$ & $23.9 \pm 2.01$ & $24.8 \pm 1.3$ & 0.41 \\
\hline Number of IVF failure (n) & $3.5 \pm 2.1$ & $4.2 \pm 1.5$ & $3.9 \pm 1.06$ & 0.34 \\
\hline Number of good quality ET (n) & $2.91 \pm 0.85$ & $2.85 \pm 0.67$ & $2.94 \pm 0.53$ & 0.54 \\
\hline $\mathrm{FSH}(\mathrm{mu} / \mathrm{ml})$ & $7.98 \pm 4.21$ & $7.22 \pm 2.98$ & $7.41 \pm 2.51$ & 0.61 \\
\hline $3^{\text {rd }}$ day Esteradiol $(\mu \mathrm{g} / \mathrm{ml})$ & $33.51 \pm 4.65$ & $35.41 \pm 2.51$ & $34.33 \pm 3.22$ & 0.32 \\
\hline
\end{tabular}

Data presented as mean \pm SD.

BMI: Body Mass Index IVF: In Vitro Fertilization

ET: Embryo Transfer

$\underline{\text { Table II. Outcome of cycles in three groups (G-CSF, saline, placebo) of RIF patients }}$

\begin{tabular}{|c|c|c|c|c|}
\hline Variables & G-CSF $(n=40)$ & Saline $(n=40)$ & Placebo $(n=20)$ & p-value \\
\hline Abortions & $2(20 \%)$ & $1(25 \%)$ & 0 & 0.06 \\
\hline Chemical pregnancy & $10(25 \%)$ & $5(12.5 \%)$ & $2(10 \%)$ & 0.04 \\
\hline Clinical pregnancy & $8 / 10(80 \%)$ & $4 / 5(80 \%)$ & $2 / 2(100 \%)$ & 0.51 \\
\hline Implantation rate & $10 / 81(12.3 \%)$ & $5 / 87(6.1 \%)$ & $2 / 42(4.7 \%)$ & 0.04 \\
\hline Fresh embryo transfer & $66(81.4 \%)$ & $77(88.5 \%)$ & $35(83.3 \%)$ & -- \\
\hline FET transfer & $15(18.5 \%)$ & $9(11.49 \%)$ & $7(16.6 \%)$ & -- \\
\hline
\end{tabular}

Data presented as n (\%).

GCSF: Granulocyte Colony Stimulating Factor $\quad$ FET: Frozen Embryo Transfer

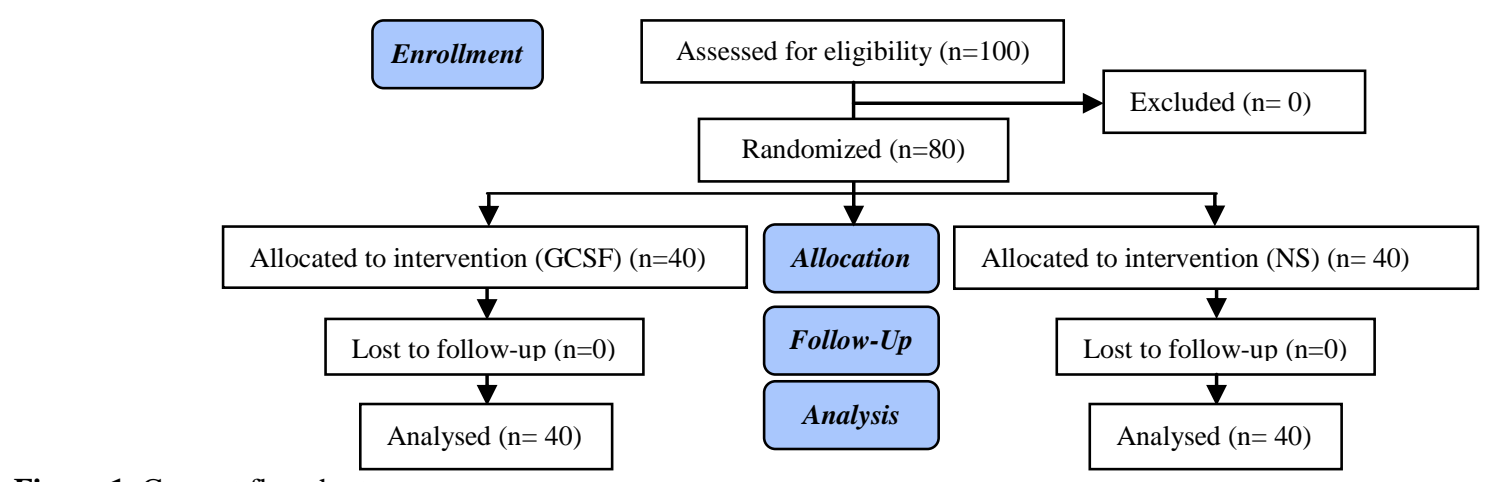

Figure 1. Consort flowchart. 


\section{Discussion}

The results of this trial suggests that intrauterine perfusion of G-CSF in RIF can increase chemical pregnancy and implantation rates however the effects on clinical pregnancy rate and abortion rate need to be evaluated. RIF may be the consequence of embryo or uterine factors. Uterine pathology like myoma, polyp, adenomyosis and adhesion can cause implantation failure; however patients with RIF have vigorously assessed for these pathologic condition and many of them have history of laparoscopic or hysteroscopic correction and evaluation of these disorders $(20,21)$.

However $40 \%$ of patients with RIF have some unrecognized lesions in endometrial cavity; in the rest there is no detectable pathology at uterine cavity (22). All eligible patients in present study have been evaluated for uterine pathology by hysteroscopy or synchronous laparoscopy. The embryo attaches to the luminal surface of the endometrium, then migrates via the luminal epithelium and invades into deep layer of endometrium which leads to implantation. Desidualization of endometrial cells is a differentiation process and it is crucial for implantation of pregnancy (2). G-CSF stimulates cellular differentiation of hemopoietic progenitor cells (23-26). The present study showed that G-CSF increases the chemical pregnancy and implantation rate in women with RIF.

In a randomized study in women with recurrent abortion $82.9 \%$ of women who were treated with G-CSF subcutaneously delivered healthy infants $(p=0.0061, O R=5.1 ; 95 \% \mathrm{Cl}$ 1.5-18.4) (27). In a randomized trial endometrial injury resulted to a significant improvement (nearly development) in the implantation and clinical pregnancy and live birth rate $(27.7 \%, 66.7 \%$ and $48.9 \%$ respectively) compared with control subjects who did not have endometrial biopsies $(28,29)$. G-CSF administration appears to be associated with an increase in regulatory $T$ cells and dendritic cells and appears to influence endometrial expression of genes which have cardinal role in implantation process (30-33).

Santjohander in 2013 showed that G-CSF in patients with recurrent miscarriage leads to better reproductive results regarding to placebo. Pregnancy rate of $47 \%$ and live birth rate of $32 \%$ was reported in G-CSF group but pregnancy rate of $27 \%(\mathrm{p}=0.016)$ and live birth rate of $14 \%(p=0.006)$ was reported in placebo group (34). Gleicher et al reported four patients with thin endometrium whom were treated with intrauterine G-CSF, except one with ectopic pregnancy, three other patients had ongoing pregnancy at the time of study report (35).

Gleicher et al reported 21 patients with thin endometrium who were treated with intrauterine G-CSF. The mean age of the patients was $40.5 \pm 6.5$ years and $76.2 \%$ of them had poor ovarian response. They had history of IVF failure $(2 \pm 2.1)$ cycles and also history of cycle cancellation due to thin endometrium (36). Endometrial thickness significantly increased and pregnancy rate was $19 \%$. They showed that G-CSF can increases endometrial thickness, but the sample size was small and there was no control group. At present study there were case group (G-CSF), control group (saline) and placebo group (without intervention) and this study showed that chemical pregnancy rates were significantly different in G-CSF group regarding to saline or placebo group.

Pregnancy outcome in 37 patients with thin endometrium $(<7 \mathrm{~mm})$ was evaluated by Kunicki. G-CSF results in improvement of endometrial thickness in both group (patients who become pregnant and patients who did not) (37). The pregnancy rate in this study was $18.9 \%$.

Li et al reported a cohort of 59 patients in FET cycle who intrauterine G-CSF during endometrial preparation were administered. Implantation rate and pregnancy rate was not significantly different (38). However the study was retrospective and the dose of G-CSF was $100 \mu \mathrm{g}$. In clinical trial of fifteen patients with history of thin endometrium who had history of cycle cancellation due to thin endometrium, GCSF intrauterine could increase endometrial thickness and 3 out of 15 patients became pregnant. The pregnancy rate was 19\% (39). Thin endometrium was resistant to other treatment, like high dose estrogen, sildnafiel, Asprin or even vit-E.

Barad et al reported that G-CSF in patients with normal endometrium and old age had no effects in pregnancy and implantation rates (40). They evaluated 141 unselected women without history of renal disease, sickle cell or 
malignancy who were undergone IVF. Seventy three of them received G-CSF and 68 received placebo. The increase in endometrial thickness was not statistically different in both groups. The clinical pregnancy and implantation rate also were not different statistically. The mean age of patients was $39.59 \mathrm{yr}$. However they believed that their results may not necessarily apply to younger patients and they declare that G-CSF losses its effectiveness in the presence of a normally proliferating endometrium, or at least when it infused intrauterine, and systemic G-CSF has different effects from local G-CSF (41).

Either G-CSF mechanisms is activation of some immunological process that are responsible for implantation or it reacts via inducing inflammation, it seems that in patients with recurrent implantation failure who have good quality embryos and uterine cavity has no polyp, myoma, adenomyosis, or adhesion or any space occupying lesion or hydrosalpinx, G-CSF may increase pregnancy and implantation rates without serious side effects (42). It seems that G-CSF can initiates a beneficial cross talk between endometrium and developing embryo and it can improve implantation through rolling, apposition, adhesion and invasion (43). This is the same stage that activated migrating leukocyte transferring vascular endothelium, where GCSF affects this process; it has to be figure out and proven.

\section{Conclusion}

G-CSF may increase pregnancy rate and implantation rate in recurrent implantation failure patients.

\section{Acknowledgements}

Deputy research of Tehran University of Medical Sciences granted this work as a research project and authors would like to appreciate their cooperation.

\section{Conflict of interest}

The authors declare that there is no conflict of interest.

\section{References}

1. Coughlan C, Ledger W, Wang Q, Liu F, Demirol A, Gurgan $\mathrm{T}$, et al. Recurrent implantation failure:
Definition and Management. Reprod Biomed Online 2014; 28: 14-38.

2. Polanski LT, Baumgarten MN, Quenby S, Brosens J, Campbell BR, Raine-Fenning NJ. What exactly do we mean by 'recurrent implantation failure'? A systematic review and opinion. Reprod Biomed Online 2014; 28: 409-423.

3. Blake D, Farquhar C, Johnson N, Proctor M. Cleavage stage versus blastocyst stage embryo transfer in assisted conception. Cochrane Database Syst Rev 2007; CD002118

4. Ferraretti AP, La Marca A, Fauser BC, Tarlatzis B, Nargund G, Gianaroli L. ESHRE consensus on the definition of 'poor response' to ovarian stimulation for in vitro fertilization: the Bologna criteria. Hum Reprod 2011; 26: 1616-1624.

5. Rotterdam ESHRE/ASRM-Sponsored PCOS Consensus Workshop Group, 2004. Revised 2003 consensus on diagnostic criteria and long-term health risks related to polycystic ovary syndrome. Fertil Steril 2004; 81: 19-25.

6. Taylor $\mathrm{H}$. The role of HOX genes in human implantation. Hum Reprod Update 2000; 6: 75-79.

7. Taylor E, Gomel V. The uterus and fertility. Fertil Steril 2008; 89: 1-16.

8. Shokeir T, El-Shafei M, Yousef H, Allam AF, Sadek E. Submucous myomas and their implications in the pregnancy rates of patients with otherwise unexplained primary infertility undergoing hysteroscopic myomectomy: a randomized matched control study. Fertil Steril 2010; 94: 724-729.

9. Bosteels J, Weyers S, Puttemans P, Panayotidis C, Van Herendael B, Gomel V, et al. The effectiveness of hysteroscopy in improving pregnancy rates in subfertile women without other gynaecological symptoms: a systematic review. Hum Reprod Update 2010; 16: 1-11.

10. Varasteh NN, Neuwirth RS, Levin B, Keltz MD. Pregnancy rates after hysteroscopic polypectomy and myomectomy in infertile women. Obstet Gynecol 1999; 94: 168-171.

11. Tremellen K, Russell P. Adenomyosis is a potential cause of recurrent implantation failure during IVF treatment. Aust N Z J Obstet Gynaecol 2011; 51: 280-283.

12. Thebault N, Broux PL, Moy L, Vialard J. Utilization of Essure micro-insert for hydrosalpinx occlusion in infertile women. J Gynecol Obstet Biol Reprod 2012; 41: $145-150$.

13. Cutting R, Morroll D, Roberts SA, Pickering S, Rutherford A. Elective single embryo transfer: guidelines for practice British Fertility Society and Association of Clinical Embryologists. Hum Fertil (Camb) 2008; 11: 131-146.

14. Yang YS, Melinda S, Ho HN, Hwang JL, Chen SU, Lin HR, et al. Effect of the number and depth of embryos transferred and unilateral or bilateral transfer in tubal embryo transfer (TET). J Assist Reprod Genet 1992; 9: 534-538.

15. Weissman A, Horowitz $E$, Ravhon A, Nahum $H$, Golan A, Levran D. Pregnancies and livebirths following ICSI with testicular spermatozoa after repeated implantation failure using ejaculated spermatozoa. Reprod Biomed Online 2008; 17: 605609.

16. Thomas J, Liu F, Link DC. Mechanisms of mobilization of hematopoietic progenitors with 
granulocyte colony-stimulating factor. Curr Opin Hematol 2002; 9: 183-189.

17. Litwin S, Lagadari M, Barrientos G, Roux ME, Margni $R$, Miranda $S$. Comparative immunohistochemical study of M-CSF and G-CSF in feto-maternal interface in a multiparity mouse model. Am J Reprod Immunol 2005; 54: 311-320.

18. Marino VJ, Roguin LP. The granulocyte colony stimulating factor (G-CSF) activates Jak/STAT and MAPK pathways in a trophoblastic cell line. J Cell Biochem 2008; 103: 1512-1523.

19. McCracken S, Layton JE, Shorter SC, Starkey PM, Barlow DH, Mardon HJ. Expression of granulocytecolony stimulating factor and its receptor is regulated during the development of the human placenta. $J$ Endocrinol 1996; 149: 249-258.

20. McCracken SA, Grant KE, MacKenzie IZ, Redman CW, Mardon HJ. Gestational regulation of granulocyte-colony stimulating factor receptor expression in the human placenta. Biol Reprod 1999; 60: 790-796.

21. Yarali $\mathrm{H}$, Bukulmez $\mathrm{O}$. The effect of intramural and subserous uterine fibroids on implantation and clinical pregnancy rates in patients having intracytoplasmic sperm injection. Arch Gynecol Obstet 2002; 266: 30-33.

22. Yasmin H, Nasir A, Noorani KJ. Hystroscopic management of Ashermans syndrome. J Pak Med Assoc 2007; 57: 553-555.

23. Yu D, Wong YM, Cheong Y, Xia E, Li TC. Asherman syndrome-one century later. Fertil Steril 2008; 89: 759-779.

24. Ayida G, Chamberlain P, Barlow D, Kennedy S. Uterine cavity assessment prior to in-vitro fertilization: comparison of transvaginal scanning, saline contrast hysterosonography and hysteroscopy. Ultrasound Obstet Gynecol 1997; 10: 59-62.

25. Daiter E, Pampfer S, Yeung YG, Barad D, Stanley ER, Pollard JW. Expression of colony-stimulating factor- 1 in the human uterus and placenta. J Clin Endocrinol Metab 1992; 74: 850-858.

26. Pampfer S, Daiter E, Barad D, Pollard JW. Expression of the colonystimulating factor-1 receptor (c-fms proto-oncogene product) in the human uterus and placenta. Biol Reprod 1992; 46: 48-57.

27. Saito S, Fukunaga R, Ichijo M, Nagata S. Expression of granulocyte colonystimulating factor and its receptor at the fetomaternal interface in murine and human pregnancy. Growth Factors 1994; 10: 135143.

28. Kauma SW, Aukerman SL, Eierman D, Turner T. Colony-stimulating factor-1 and c-fms expression in human endometrial tissues and placenta during the menstrual cycle and early pregnancy. J Clin Endocrinol Metab 1991; 73: 746-751.

29. Scarpellini F, Sbracia M. Use of granulocyte colonystimulating factor for the treatment of unexplained recurrent miscarriage: a randomised controlled trial. Hum Reprod 2009; 24: 2703-2708.

30. Barash A, Dekel N, Fieldust S, Segal I, Schechtman E, Granot I. Local injury to the endometrium doubles the incidence of successful pregnancies in patients undergoing in vitro fertilization. Fertil Steril 2003; 79: 1317-1322.

31. Zhou L, Li R, Wang R, Huang HX, Zhong K. Local injury to the endometrium in controlled ovarian hyperstimulation cycles improves implantation rates. Fertil Steril 2008; 89: 1166-1176.

32. Würfel W, Santjohanser C, Hirv K, Bühl M, Meri O, Laubert I, et al. High pregnancy rates with administration of granulocyte colony-stimulating factor in ART-patients with repetitive implantation failure and lacking killer-cell immunoglobulin-like receptors. Hum Reprod 2010; 25: 2151-2152.

33. Rahmati $M$, Petitbarat $M$, Dubanchet $S$, Bensussan A, Chaouat G, Ledee N. Granulocyte-Colony Stimulating Factor Related Pathways Tested on an Endometrial Ex-Vivo Model. PloS One 2014; 9: e102286

34. Santjohanser C, Knieper C, Franz C, Hirv K, Meri O, Schleyer M, et al. Granulocyte-colony stimulating factor as treatment option in patients with recurrent miscarriage. Arch Immunol Ther Exp (Warsz) 2013; 61: 159-164.

35. Gleicher N, Vidali A, Barad DH. Successful treatment of unresponsive thin endometrium. Fertil Steril 2011; 95: 13-17.

36. Gleicher N, Kim A, Michaeli T, Lee HJ, Shohat-Tal A, Lazzaroni $\mathrm{E}$, et al. A pilot cohort study of granulocyte colony-stimulating factor in the treatment of unresponsive thin endometrium resistant to standard therapies. Hum Reprod 2013; 28: 172- 177.

37. Kunicki M, Łukaszuk K, Woclawek-Potocka I, Liss J, Kulwikowska $P$, Szczyptańska J. Evaluation of granulocyte colony-stimulating factor effects on treatment-resistant thin endometrium in women undergoing in vitro fertilization. Biomed Res Int 2014: 913235

38. Li Y, Pan P, Chen X, Li L, Li Y, Yang D. Granulocyte colony-stimulating factor administration for infertile women with thin endometrium in frozen embryo transfer program. Reprod Sci 2014; 21: 381-385.

39. Tehraninejad E, Davari Tanha F, Asadi E, Kamali K, Aziminikoo E, Rezayof E. G-CSF Intrauterine for Thin Endometrium, and Pregnancy Outcome. J Fam Reprod Health 2015; 9: 107-112.

40. Barad DH, Yu Y, Kushnir VA, Shohat-Tal A, Lazzaroni $\mathrm{E}$, Lee $\mathrm{HJ}$, et al. A randomized clinical trial of endometrial perfusion with granulocyte colonystimulating factor in in vitro fertilization cycles: impact on endometrial thickness and clinical pregnancy rates. Fertil Steril 2014; 101: 710-717.

41. Scarpellini F, Sbracia M. Use of granulocyte colonystimulating factor for the treatment of unexplained recurrent miscarriage: a randomised controlled trial. Hum Reprod 2009; 24: 2703-2708.

42. Bildirici I, Bukulmez O, Ensari A, Yarali $H$, Gurgan $T$. A prospective evaluation of the effect of salpingectomy on endometrial receptivity in cases of women with communicating hydrosalpinges. Hum Reprod 2001; 16: 2422-2426.

43. Genbacev O, Prakobphol A, Foulk R, Krtolica A, llic $\mathrm{D}$, et al. Trophoblast L-selectin-mediated adhesion at the maternal-fetal interface. Science 2003; 299: 405408 